\title{
Cellulose on Miscibility and Thermal Studies of Acetate and Modified Industrial Waste Lignin Blends
}

\author{
Ashok Nerella \\ M. Sc (Organic Chemistry), Lecturer in Chemistry, DR. BR. Ambedkar GMR Polytechnic for Women, Karimnagar
}

\begin{abstract}
An industrial waste lignin was modified by esterification using acetic acid. The modified lignin (ML) was blended upto $50 \mathrm{wt} \%$ with cellulose acetate. The blends were prepared by solution blending method. The thermal properties and miscibility of cellulose acetate (CA) and modified lignin (ML) blends were investigated by thermo gravimetric analysis (TGA), differential scanning calorimetry (DSC), scanning electron microscopy (SEM) and Fourier transform infra-red spectroscopy (FTIR) over the entire range of composition. Although the modified lignin shifts the onset of CA decomposition to lower temperatures, the CA/ML blends are thermally more stable than CA over the wider temperature range. The TGA results suggest all blends are compatible. These results correlate well with the glass transition temperature $(\mathrm{Tg})$ data where single $\mathrm{Tg}$ has been obtained for all these blends. There is a slight decrease in $\mathrm{Tg}$ as the lignin wt\% increase in blends, where in (70/30) composition shows higher value than CA. No phase separation was found in SEM images of blends. The FTIR analysis revealed the formation of blending between cellulose acetate and modified lignin.
\end{abstract}

Keywords: Cellulose acetate, Modified lignin, Thermal stability, DSC, SEM, FTIR.

\section{INTRODUCTION}

Blending of polymers is one of the simplest means to obtain a variety of physical and chemical properties from the constituent polymers. The gain in newer properties depends on the degree of compatibility or miscibility of polymers at molecular level (Guru et al, 2012). Utilization of biodegradable/ biobased material is receiving increasing attention as the world looks to find alternatives to petrochemicals. Traditionally, lignins have been incorporated into various polymeric materials as low cost filler (Kubo and Kadla, 2004). Lignin exists in plant cell wall and second only to cellulose in overall natural abundance. Lignin is a complex, amorphous, polyphenolic, natural organic polymeric material and is built up from poly phenyl propane units. Enormous quantity of industrial lignin is produced as a byproduct of papermaking. Kraft/ alkaline and sulphite process are the two main delignification processes used in pulping industry, thus lignin removed from wood is available in large quantities as a waste from pulp mills (Mankar and Chaudhari, 2012). Depending on the type and length of chemical processing, the lignins vary in molecular weight, functional groups present, degree of condensation, types of inter monomeric linkages and the type and ratio of monomeric units (Kubo and Kadla, 2004).

The effect of poly (ethylene oxide) molecular mass on miscibility and hydrogen bonding with lignin was studied by (Kubo and Kadla, 2006) using thermal analysis, FTIR and DSC analysis which revealed miscibility of blends over the entire blend ratio. (Pucciariello et al., 2004) used the lignin powder, obtained from an abundant and low cost source, straw for the preparation of blends with low density polyethylene (LEDP), high density polyethylene (HDPE) and atactic polystyrene (PS). The obtained blends are processable through the conventional techniques used for thermoplastics. The modulus slightly increases for most lignin-polymer blends, while the tensile stress and elongation reduced. Moreover lignin acts as stabilizer against UV radiation for all blends.

(Fan et al., 2011) prepared a new biodegradable blend by incorporating alkaline lignin (AL) filler into the poly (butylene succinate) as a matrix. The blends were prepared between the 5-30\% weight percentages of alkaline lignin. The mixture was compression-moulded into a sheet and their structural and mechanical properties were studied. (Vengal and Srikumar, 2005) extracted the lignin from waste wood chips of paper industry and blended with established biopolymers like starch and gelatin. They tested the water absorption and tensile strength of different lignin-starch lignin-gelatin and starch-gelatin-lignin polymer films. Solution blending followed by curing was the method adopted for the preparation of films. A literature survey reveals that lignin was blended with various polymers like poly (vinyl alcohol), poly (ethylene oxide), poly (ethylene perphthalate), poly (propylene) and their thermal properties, chemical structure and molecular interactions were studied ( Kadla and Kubo; 2004, Kubo and Kadla; 2003, Kadla and Kubo; 2003, Gowarikar et al. 1986).

The properties of lignin-based blends and composites can be improved by appropriate chemical modifications aimed 
at optimizing the interactions between or among the components of these materials, essentially by reducing the corresponding interfacial energy and thus enhancing their compatibility. To increase solubility and compatibility of polar lignin towards the non-polar polymers, number of workers modified the lignin to decrease its polarity for different applications ( $\mathrm{Li}$ and Sarkanen, 2005). The acetosolv lignin from sugarcane bagasse was treated for hydroxymethylation and studied by FTIR analysis (Benar and Gonclaves, 1999).

(Maldhure et al. 2011) achieved the microwave assisted chemical modification of lignin through esterification using maleic anhydride. This modified lignin was blended in different proportions upto 25 mass $\%$ with polypropylene by mixing the melts in Brabender Electronic Plasticoder at $190{ }^{\circ} \mathrm{C}$. The thermal and structural properties of blends were investigated by TG, DSC, WAXD and SEM analysis. The thermal stability of blends was found to increase due to antioxidant property of modified lignin.

(Thielemans et al., 2005) esterified the kraft lignins from hardwood and softwood with several anhydrides to alter their solubility behavior in non-polar solvents, such as styrene containing thermoset resins. (Zhong et al., 2011) alkylated the lignin with bromododecane which improved compatibility with polypropylene and enabled upto 70 wt\% lignin to be incorporated into thermally stable composites.

(Ghosh et al., 2000) prepared thermoplastic blends of several biodegradable polymers like cellulose acetate butyrate, a starch-caprolactone copolymer and poly (hydroxybutyrate) with lignin esters.

Cellulose acetate is a plastic produced by the action of acetic anhydride on cellulose in presence of either sulphuric acid or perchloric acid as a catalyst (Seymour, 1971). It can be used for great variety of applications (eg. for films, membrane or fibers) (Fischer et al., 2008).

Various researchers blended the cellulose acetate with some polymers by solution casting method. (Meenakshi et al., 2002) studied the dried Musa paradiciaca (banana) stem and veins of the leaves as a starting material for the production of cellulose and cellulose acetate was mixed with polystyrene to form blend of cellulose acetatepolystyrene in order to provide enhanced stability and extended utility to the end products.

(Rials and Glasser, 1989 a) prepared the blends of hydroxypropyl cellulose (HPC) and organosolv lignin (OSL) by mixing the solutions of both pyridine and dioxane and casted films. They also prepared the blends by melt extrusion and studied its mechanical properties, SEM, DSC, WAXD and DMTA. Similarly, they prepared blends of lignin with ethyl cellulose (EC) and cellulose acetate/ butyrate $(\mathrm{CAB})$ by solution casting from dioxane and studied the dynamic mechanical analysis (DMTA) and DSC analysis (Rials and Glasser, 1989 b).
In the present study industrial waste lignin has been modified by esterification and blended with cellulose acetate by solution blending (Gowarikar et al.1986, Seymour et al. 1971). The solid state miscibility was studied by analyzing DSC and SEM of blends. The thermal degradation behaviors of the blends were studied by thermogravimetric analysis. The molecular interaction and bonding was investigated by FTIR.

\section{MATERIALS AND METHODS}

\subsection{Cellulose acetate}

CA was obtained from Lobachemie. Its Melting point is $240{ }^{\circ} \mathrm{C}$, acetyl content is $29-45 \%$, Mol. Wt. is 316.39 , density is $1.3 \mathrm{~g} / \mathrm{ml}$ at $25^{\circ} \mathrm{C}$, and refractive index is 1.475 .

\subsection{Lignin extraction}

Lignin was precipitated from black liquor using $20 \%$ sulphuric acid. The precipitate obtained was thoroughly washed with water and filtered. The residue was dried and weighed. The crude lignin was purified using 1, 4 dioxane.

\subsection{Esterification of lignin}

Esterification of lignin was done by Fischer Esterification method. $10 \mathrm{gm}$ of pure lignin was dissolved in $100 \mathrm{ml}$ of ethanol and transferred in RB flask. In this solution $30 \mathrm{ml}$ of acetic acid was added and to this mixture 10-12 drops of conc. sulphuric acid was added by swirling the flask. The reaction mixture was refluxed for one and half hour by using water condenser. During refluxation RB flask was kept in water bath. After completion of reaction the flask was allowed cooling at room temperature. The cooled mixture was then poured in large quantity of distilled water and filtered. The residue is the lignin derivative which was dried at room temperature (Marchetti and Errazu; 2008, Williamson, 1994). The reaction scheme of esterification of lignin is given in scheme 1 .
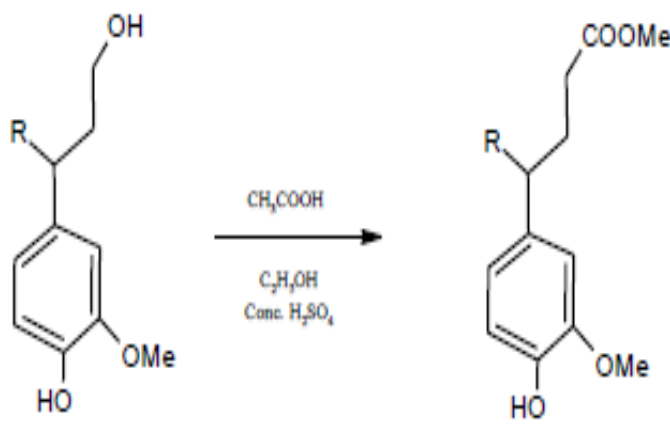

Fig1 . Reaction scheme for esterification of lignin by acetic acid

\section{BLEND PREPARATION}

The CA/ML blends were prepared by mixing the individual solutions (wt \%) of components in 1, 4 dioxane. The components were dissolved in the solvent. The solutions were mixed together and stirred for approximately half an hour. The resulting solution mix 
was poured in small installments in large quantity of respectively. The results show that the thermal stability of distilled water (non-solvent of CA and ML). There is a blends increases with increase in ML\%. Similar kinds of formation of precipitate of blend in water, which was then results were obtained by (Mousavioun et al., 2010) for immediately pick out from water with the help of glass rod poly (hydroxybutyrate) and soda lignin blends. This may and placed on filter paper. The blends were dried in air at be due to intermolecular interactions between cellulose room temperature for 4-5 days. The dried blends then acetate and modified lignin. The decrease in weight loss crushed to fine powder with the help of mortar and pestle. with increase in ML content in blends indicates that blends It was then used for further analysis. The composition of are thermally more stable than CA in wider temperature blends obtained by mixing wt $\%$ solutions of cellulose range.

acetate and modified lignin is given in Table1.

\section{ANALYSIS}

\subsection{Thermogravimetric analysis (TGA)}

The thermal decomposition studies of CA, ML and CAML blends were carried out in TA instrument Dimond TG/DTA (Perkin Elmer, USA). Heating was at a rate of $10^{\circ} \mathrm{C} / \mathrm{min}$. and was performed from $25^{\circ} \mathrm{C}$ to $820^{\circ} \mathrm{C}$, in an atmosphere of nitrogen.

\subsection{Differential Scanning Calorimetry (DSC)}

The DSC scans of CA, ML and CA-ML blends were collected in NETZSCH DSC 204 instrument heated from $25^{\circ} \mathrm{C}$ to $350^{\circ} \mathrm{C}$ at a heating rate $10^{\circ} \mathrm{C} / \mathrm{min}$ in nitrogen atmosphere.

\subsection{Scanning electron microscopy (SEM)}

The morphology of CA, ML and CA-ML blends were examined using scanning electron microscope, type JEOL Model JSM-6390LV at an accelerating voltage $20 \mathrm{kV}$ with SEI image mode and $1 \mu \mathrm{m}$ resolution.

\subsection{Fourier transform infra-red spectroscopy (FTIR)} IR spectra of Lignin, ML , CA and CA-ML (50/50) blend were collected using Nicolet MAGNA 550 (Nicolet Instrument Corporation, USA) in a spectral range 4000$400 \mathrm{~cm}-1$ using 64 scans at $4 \mathrm{~cm}-1$ resolution.

\section{RESULT AND DISCUSSION}

\section{Thermo gravimetric analysis}

Thermal stability of CA, ML and CA/ML blends were assessed. The TGA thermogram for $\mathrm{CA}$ and $\mathrm{CA} / \mathrm{ML}$ blends are shown in Fig.1. The effect of ML in different proportions on thermal stability of CA/ML blends was studied by means of thermo gravimetric experiments. Table.2 shows the thermo gravimetric results corresponding to onset, offset, midpoint and residual mass $\%$ obtained at $830{ }^{\circ} \mathrm{C}$ of pure lignin, ML, CA and $\mathrm{CA} / \mathrm{ML}$ blends. It was observed that there is increase in thermal stability of blends as compared to CA. The thermogram (Fig.1) indicates that, decomposition temperature decrease slightely in blends as compared to CA. Cellulose acetate as well as all the blends shows single stage degradation. The degradation of modified lignin starts at very $250^{\circ} \mathrm{C}$ and produces remarkable char residues. The weight loss percentage of $\mathrm{CA}$ at 820 is found to be $95.1 \%$, whereas CA/ML blends $90 / 10,70 / 30$ and $50 / 50$ composition were $99.6 \%, 89.3 \%$ and $78.7 \%$
The TGA analysis shows remarkable increase in thermal stability for $\mathrm{CA} / \mathrm{ML}$ blends. In general, the thermal degradation temperature of $(50 / 50)$ blend is equivalent to CA whereas (70/30) and (90/10) shows slight decrease in degradation temperatures as compared to CA.

\section{Differential scanning calorimetric analysis}

The most accepted parameter to assess polymer miscibility is the $\mathrm{Tg}$. A single $\mathrm{Tg}$ of blend implies complete miscibility between the polymer pairs in their amorphous fractions. Fig. 2 presents the DSC curves of ML, CA and $\mathrm{CA} / \mathrm{ML}$ blends. The Tg was taken as midpoint of the change of slopes in DSC curves. Fig.2 illustrates the Tg of modified lignin is at $68.39^{\circ} \mathrm{C}$. The $\mathrm{Tg}$ of CA was found to be at $52.39{ }^{\circ} \mathrm{C}$, which shows correlation with literature data ( Mark et al., 1948).

According to DSC curve all CA/ML blends show single $\mathrm{Tg}$ at $42.39^{\circ} \mathrm{C}, 53.39^{\circ} \mathrm{C}$ and $48.39^{\circ} \mathrm{C}$ for $(90 / 10),(70 / 30)$ and $(50 / 50)$ blends respectively. The single $\mathrm{Tg}$ is the indication that all blends are miscible. It was observed that (90/10) and (50/50) blend composition show lower $\mathrm{Tg}$ than CA but the $\mathrm{Tg}$ of $(70 / 30)$ blend is $53.39^{\circ} \mathrm{C}$, which is higher than $\mathrm{Tg}$ value of CA. Increase in $\mathrm{Tg}$ with increase in content of lignin has been found by (Rials and Glasser, 1989 a) for hydroxypropyl cellulose-lignin blends.

\section{Scanning electron microscopy analysis}

SEM analysis is the powerful technique to determine miscibility of blends. In order to further investigate the interaction between ML and CA in the blend we have carried out the morphological investigations by SEM analysis. The SEM micrographs of lignin, modified lignin, cellulose acetate and CA/ML blends are shown in Fig.3 (a, $b, c, d, e$ and $f$ ). The SEM image of modified lignin is very much different from that of lignin. In case of blends no phase separation has been observed. All the blends are homogeneously mixed together. Thus the SEM data follow similar trends as the data obtained from the $\mathrm{Tg}$ of blends.

\section{Fourier transformed infrared spectroscopy}

Purification of lignin was done before its modification. Modification of lignin was carried out by esterification using acetic acid in presence of conc. H2SO4. The reaction takes 1 and $1 / 2 \mathrm{hrs}$ for complete esterification. The chemical change occurred during esterification were determined by FTIR. Fig. 4 shows the FTIR spectra of lignin and modified lignin (ML). 
Vol. 4, Issue 2, February 2017

The lignin spectra show absorbance at $3424.7 \mathrm{~cm}-1$ for stretching of -OH group. The absorbance at $2934.4 \mathrm{~cm}-1$ arises from $\mathrm{C}-\mathrm{H}$ stretching in methyl/ methylene group. The small peak at $2847.8 \mathrm{~cm}-1$ attributed to $\mathrm{C}-\mathrm{H}$ stretching. The absorbance at $1717.3 \mathrm{~cm}-1$ for $\mathrm{C}=\mathrm{O}$ stretching in $\mathrm{COOH}$. The peak occurred at $1267.9 \mathrm{~cm}-1$ is for $\mathrm{C}=\mathrm{O}$ stretching in esters. The peaks at $1513.5 \mathrm{~cm}-1$ and $1456.7 \mathrm{~cm}-1$ are for aromatic skeletal vibrations. C-H deformation in $\mathrm{CH} 3$ was found at $1367.1 \mathrm{~cm}-1.1328 .0$ $\mathrm{cm}-1$ shows syringyl ring breathing with $\mathrm{C}=\mathrm{O}$ stretching. The peaks at $1112.73 \mathrm{~cm}-1$ and $1031.72 \mathrm{~cm}-1$ corresponds to $\mathrm{C}-\mathrm{H}$ in plane deformation

The modified lignin shows a broad peak at $3436.1 \mathrm{~cm}-1$ for hydroxyl group stretching. The peaks at 2958.7, 2850.2 cm-1 show C-H stretching in methyl/methylene group. The absorbance at $1604.2,1510.0$ and $1455.3 \mathrm{~cm}-1$ corresponds to aromatic skeletal vibrations. The lignin acetate shows increase in the intensity of band at 1715.8 $\mathrm{cm}-1$ and $1217.6 \mathrm{~cm}-1$ for $\mathrm{C}=\mathrm{O}$ stretching in esters. The strong peaks observed at $1111.4 \mathrm{~cm}-1$ and $1035.2 \mathrm{~cm}-1$ assigned to aromatic $\mathrm{C}-\mathrm{H}$ in plane deformation guaiacyl type. Absorbance at $836.6 \mathrm{~cm}-1$ and $634.8 \mathrm{~cm}-1$ shows $\mathrm{C}$ $\mathrm{H}$ deformation. Increase in relative peak $1715.8 \mathrm{~cm}-1$ is attributed to esterification of pure lignin. It has been observed that the intensity of absobance for few peaks is increased in spectra of modified lignin than lignin ( Boeriu et al. 2004; Sharma,2000; Ibrra, 2005, Sun, 2003).

The IR spectra of CA, ML and CA-ML (50/50) blend are shown in the Fig.5. The peak at $2866 \mathrm{~cm}-1$ is attributed to $\mathrm{C}-\mathrm{H}$ stretching in methylene a group has been found in CA-ML (50/50) blend. The same peak was observed at $2850 \mathrm{~cm}-1$ in ML but not in CA. The peak at $1639 \mathrm{~cm}-1$ observed in CA shifted to $1604 \mathrm{~cm}-1$ in CA-ML (50/50) blend which is assign to $\mathrm{C}=\mathrm{C}$ stretching in aromatics. The peaks found at $1510 \mathrm{~cm}-1$ and $1512 \mathrm{~cm}-1$ attributed to aromatic skeletal vibrations present in ML and CA-ML $(50 / 50)$ blend respectively. The same peak was not obtained in CA. The intensity at $1455 \mathrm{~cm}-1$ assign to $\mathrm{C}=\mathrm{C}$ stretching in aromatic compound has been lowered in $\mathrm{CA}$ ML (50/50) blend as compared to ML. the peaks obtained at $1375 \mathrm{~cm}-1$ and $1378 \mathrm{~cm}-1$ assign to $\mathrm{C}-\mathrm{H}$ deformation in $\mathrm{CH} 2 / \mathrm{CH} 3$ shown by CA-ML (50/50) blend and CA.

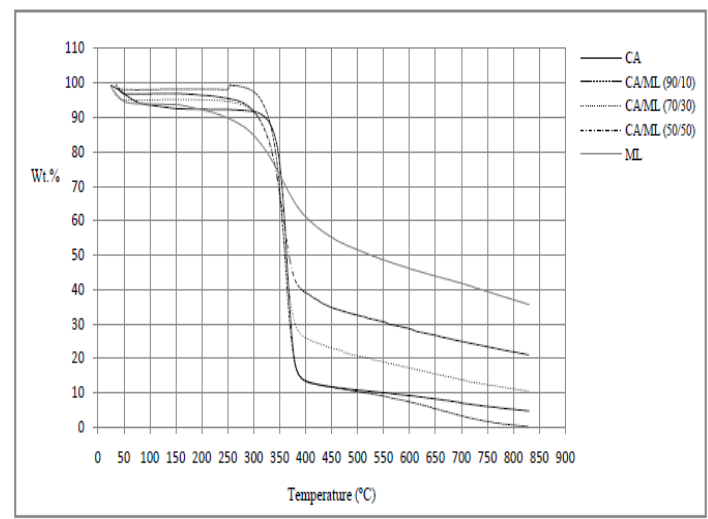

Fig.1 Thermo gravimetric analysis of CA, ML and $\mathrm{CA} / \mathrm{ML}$ blends
The intensity of peak has been increased in CA-ML (50/50) blend whereas the peak was absent in ML. The intensity of peak at $1047 \mathrm{~cm}-1$ attributed to C-O stretching in esters has been increased in CA-ML (50/50) blend as compared to $\mathrm{CA}$ and $\mathrm{ML}$

\section{CONCLUSION}

The esterification (modification) of lignin has been confiremed through FTIR analysis. The miscibility of modified lignin cellulose acetate blends has been analysed using thermal analysis, FTIR and SEM. The modified lignin improved the overall thermal stability of cellulose acetate, though it reduced the initial temperature of decomposition of cellulose acetate.

The CA/ML blend of 70/30 composition shows higher $\mathrm{Tg}$, while 90/10 and 50/50 blend composition show lower $\mathrm{Tg}$ than CA. Results obtained help to clarify the interaction between modified lignin and cellulose matrix, mainly in the degradation process of pure lignin has an influence on the degradation process of polymer matrix. The SEM micrographs of blends do not show any phase separation. The intermolecular interactions were confirmed between ML and CA by FTIR analysis.

\section{REFERENCES}

[1] Guru GS., Prasad P, Shivakumar HR, Rai SK. (2012): Miscibility and thermal and mechanical studies of methylcellulose/ poly (vinyl alcohol) blends. Int. J. Res. Pharm.Chem., 2(4): 957- 968.

[2] Kubo S, Kadla JF (2004): Poly (ethylene oxide)/ organosolve lignin blends: relationship between thermal properties, chemical structure and blend behavior. Macromolecules, 37:6904-6911.

[3] Mankar SS, Chaudhari AR (2012): Industrial waste lignin- a promising biomaterial. Bionano Frontier, 5:337-341.

[4] Kubo S, Kadla JF. (2006): Effect of poly (ethylene oxide) molecular mass on miscibility and hydrogen bonding with lignin. Holzforschung, 60:245-252.

[5] Pucciariello R, Villani V, Bonini C, D’Auria M, Vetere T. (2004): Physical properties of straw lignin-based polymer blends. Polymer, 45: 4159-4169.

[6] Fan D, Chang PR, Lin N, Yu J, Huang J. (2011): Structure and properties of alkaline lignin filled poly (butylenes succinate) plastics. Iranian Polymer Journal, 20 (1): 3-14.

[7] Vengal JC, Srikumar M. (2005): Processing and study of novel lignin-starch and lignin-gelatin biodegradable polymeric films. Trends Biomater. Artif. Organs, 18(2): 237-234.

[8] Kadla JF, Kubo S. (2004): Lignin based polymer blends: analysis of intermolecular interactions in lignin- synthetic polymer blends. Composites: Part A, 35: 395- 400.

[9] Kubo S, Kadla JF. (2003): The formation of strong intermolecular interactions in miscible blends of poly (vinyl alcohol) and lignin. Biomacromolecules, 4: 561-567.

[10] Kadla JF, Kubo S. (2003): Miscibility and hydrogen bonding in blends of poly (ethylene oxide) and kraft lignin. Macromolecules, 36: 7803-7811.

[11] Gowariker VR, Viswanathan NV, Sreedhar (1986):Polymer Science, New Age International (P) Limited, New Delhi, India,

[12] Li Y, Sarkanen S. (2005): Miscible blends of kraft ligninderivatives with low-Tg polymers. Macromolecules, 38: 2296-2306.

[13] Benar P, Gonclaves AR. (1999): Principal component analysis of the hydroxymethylation of sugarcane lignin: a time depending study by FTIR. J. Wood Chem. Technol.19 (1\&2): 151-165.

[14] Maldhure AV, Chaudhari AR, Ekhe JD. (2011): Thermal and structural studies of polypropylene blended with esterified industrial waste lignin. J. Therm. Anal. Cal., 103: 625- 632. 
[15] Thielemans W, Wool RP. (2005): Lignin esters for use in unsaturated thermosets: lignin modification and solubility modeling. Biomacromolecules, 6: 1895-1905.

[16] Zhong M, Dai H, Yao H, Dai D, Zhou Y, Yang J, Chen F. Strongflexible high-lignin polypropylene blends, Society of Plastic Engineers (Plastic research online) 10.1002/spepro 003642.

[17] Ghosh I, Jain RK, Glasser WG. (2000): Blends of biodegradable thermoplastics with lignin etsrs. ACS Symposium Series, 742: 331350 .

[18] Seymour RB. (1971): Introduction to polymer chemistry, McGrawHill Book Company, New York, London.

[19] Fischer S, Thümmeler K, Volkert B, Hettrich K, Scemidt I, Fischer K. (2008): Properties and applications of cellulose acetate. Macromolecule Symposium, 262: 89-96.

[20] Meenakshi P, Noorjahan SE, Rajini R, Venkateswarlu U, Rose C, Sastry TP. (2002): Mechanical and microstructure studies on the modification of CA film by blending with PS. Bull. Mater. Sci., 25: 25-29.

[21] Rials TG, Glasser WG. (1989a): Multiphase materials with lignin. IV blends of hydroxypropyl cellulose with lignin. J. Appl. Polym.Sci., 37: 2399-2415

[22] Rials TG, Glasser WG. (1989b): Multiphase materials with lignin. VI effect of cellulose derivative structure on blend morphology with lignin. Wood Fiber Sci., 21(1): 80-90.

[23] Marchetti JM, Errazu AF. (2008): Esterification of free fatty acids using sulphuric acid as catalyst in the presence of triglycerides. Biomass and Bioenergy, 32: 892-895.

[24] Williamson KL. (1994): Macroscale and microscale organic experiments, 2nd Ed. Houghton Mifflin, Boston, 385.

[25] Mousavioun P, Doherty WOS, George G. (2010): Thermal stability and miscibility of poly (hydroxybutyrate) and soda lignin blends. Ind. Crop. Prod., 31: 52-58.

[26] Mark H, Proskauer ES, Doty PM, Frilette VJ, Zimm BH. (1948): The Science of Plastics, Interscience Publisher, Inc., New York, pp.196.

[27] Boeriu G, Bravo D, Gosselink RJA,. Van Dam JEG. (2004): Characterization of structure-dependent functional properties of lignin with infrared spectroscopy. Ind.Crop. Prod. 20:205-218.

[28] Sharma YR. (2000): Elementary Organic Spectroscopy, S. Chand and Company, New Delhi, pp. 69- 150.

[29] Ibarra D, José C, del Río, Gutiérrez A, Rodríguez IM, Romero J, Martínez MJ, Martínez AT. (2005): Chemical characterization of residual lignins from eucalyptus paper pulps. J. Anal. Appl. Pyrolysis, 74: 116-122.

[30] Sun JX, Sun XF, Sun RC, Flower P, Baird M. (2003): Inhomogeneities in the chemical structure of sugarcane bagasse lignin. J. Agric. Food Chem., 51(23): 6719-6725 